Revista Brasil. Bot., V.33, n.1, p.143-153, jan.-mar. 2010

\title{
Modelos preditores da fitomassa aérea da Floresta Baixa de Restinga
}

\author{
DÉBORAH MOREIRA-BURGER ${ }^{1,2}$ e WELINGTON BRAZ CARVALHO DELITTI ${ }^{1}$
}

(recebido: 11 de dezembro de 2008; aceito: 11 de fevereiro de 2010)

\begin{abstract}
Regression models to estimate aboveground phytomass of the Floresta Baixa de Restinga). The purpose of this study was to develop equations to estimate the aboveground phytomass of the Floresta Baixa de Restinga. We selected 102 trees of 29 species occurring in the study area and 102 trees of Syagrus romanzoffiana (Cham.) Glassman, distributed proportionately among the diameter classes of trees. The trees were cut at ground level and the total height and diameter at breast height $(\mathrm{DBH})$ of each tree were measured. Leaves and woody material were separated in order to determine their fresh weights under field conditions. Samples of each fraction were oven dried at $70^{\circ} \mathrm{C}$ to constant weight to determine their dry weight. The models were developed by using single linear regression analysis, where the dependent variable was the dry mass (DW), and the independent variables were height (h), diameter (d), the product of diameter and height $\left(\mathrm{d}^{2} \mathrm{~h}\right)$ and the product of diameter, height and woody density $\left(\rho \mathrm{d}^{2} \mathrm{~h}\right)$. The models developed indicate that the diameter explains much of the variability of the trees phytomass and the tree height is the explanatory variable of the specific equation for Syagrus romanzoffiana. The best equations to estimate aboveground phytomass were: $\ln \mathrm{DW}=-1.352+2.009 \ln \mathrm{d}\left(\mathrm{R}^{2}=0.96 ; s_{y x}=0.34\right)$ for the community without Syagrus, $\ln \mathrm{DW}=-2.052+0.801 \mathrm{ln} \mathrm{d}^{2} \mathrm{~h}\left(\mathrm{R}^{2}=0.94 ; s_{y x}=0.38\right)$ for the community with Syagrus and $\ln \mathrm{MS}(\mathrm{kg})=-0.884+$ $2.40 \ln \mathrm{h}\left(\mathrm{R}^{2}=0.92 ; s_{y x}=0.49\right)$ for Syagrus.
\end{abstract}

Key words - Atlantic Forest, allometry, biomass, forest biomass estimation, Syagrus romanzoffiana

RESUMO - (Modelos preditores da fitomassa aérea da Floresta Baixa de Restinga). Este estudo teve como objetivo desenvolver modelos preditores de fitomassa epigéa da vegetação arbórea da Floresta Baixa de Restinga. Foram selecionadas 102 árvores de 29 espécies ocorrentes na área de estudo e 102 indivíduos de jerivá (Syagrus romanzoffiana (Cham.) Glassman), distribuídos proporcionalmente entre as classes de diâmetro da vegetação arbórea. As árvores foram cortadas, ao nível do solo e foram medidos a altura total e o diâmetro à altura do peito (DAP) de cada árvore. As folhas foram separadas do lenho e a massa fresca da porção lenhosa e foliar medidas separadamente. Amostras de cada fração foram secas a $70{ }^{\circ} \mathrm{C}$, até peso constante, para determinação da massa seca das árvores. Os modelos foram desenvolvidos através de análise de regressão linear, sendo a variável dependente a massa seca (MS) das árvores e as variáveis independentes a altura (h), o diâmetro a altura do peito (d) e as relações $\mathrm{d}^{2} \mathrm{~h}$ e $\mathrm{d}^{2} \mathrm{~h}$ multiplicada pela densidade da madeira $\left(\rho \mathrm{d}^{2} \mathrm{~h}\right)$. Os modelos desenvolvidos indicam que o diâmetro explica grande parte da variabilidade da fitomassa das árvores da restinga e a altura é a variável explanatória da equação específica para o jerivá. Os modelos selecionados foram: $\ln \mathrm{MS}(\mathrm{kg})=-1,352+2,009 \ln \mathrm{d}\left(\mathrm{R}^{2}=0,96 ; s_{y x}=0,34\right)$ para a comunidade vegetal sem jerivá, $\ln \mathrm{MS}(\mathrm{kg})=-2,052+0,801 \ln \mathrm{d}^{2} \mathrm{~h}\left(\mathrm{R}^{2}=0,94 ; s_{y x}=0,38\right)$ para a comunidade incluindo o jerivá, e ln $\mathrm{MS}(\mathrm{kg})=-0,884+$ $2,40 \ln \mathrm{h}\left(\mathrm{R}^{2}=0,92 ; s_{y x}=0,49\right)$ para o jerivá.

Palavras-chave - alometria, biomassa, estimativa de biomassa florestal, Floresta Atlântica, Syagrus romanzoffiana

\section{Introdução}

A biomassa é considerada um importante parâmetro de caracterização dos ecossistemas, indicando sua capacidade de acúmulo de matéria orgânica ao longo do tempo. Em condições ideais, um ecossistema maduro atinge seu potencial máximo de armazenamento de material e os valores de biomassa podem ser usados como um ponto de referência.

A matéria orgânica acumulada pela vegetação, a fitomassa, pode ser determinada através do corte e medida da massa das plantas de uma área amostral relativamente

1. Universidade de São Paulo, Departamento de Ecologia do Instituto de Biociências, Rua do Matão, Travessa 14, 321, 05508-900 São Paulo, SP, Brasil.

2. Autor para correspondência: deborahmburger@yahoo.com.br pequena, cujo valor médio encontrado é extrapolado para uma área maior. Ecossistemas florestais raramente têm sua fitomassa medida por métodos destrutivos devido às dificuldades inerentes a este tipo de estudo, como o elevado custo e as restrições legais referentes ao corte da vegetação. A fitomassa de florestas, em geral, é estimada de maneira indireta, através da aplicação de modelos preditores desenvolvidos a partir das relações alométricas encontradas nos componentes da vegetação. Porém, são necessários modelos específicos para os diferentes ecossistemas para que o erro das estimativas seja minimizado (Rochow 1974, McWillian et al. 1993).

Os modelos preditores são elaborados a partir de análise de regressão, onde se estudam as relações entre a massa seca das árvores e seus dados facilmente mensuráveis, como altura e diâmetro. Diversos tipos 
de modelos de regressão e diferentes combinações das variáveis têm sido usados no desenvolvimento de modelos preditores de fitomassa para ecossistemas tropicais. No Brasil os modelos preditores de fitomassa florestal, a partir de métodos destrutivos, concentraramse nas Florestas Amazônicas (Klinge et al. 1975, Uhl \& Jordan 1984, Uhl et al. 1988, McWillian et al. 1993, Kauffman et al. 1994, Santos 1996, Higuchi et al. 1998, Nelson et al. 1999, Nogueira et al. 2007, Nogueira et al. 2008). Em menor número, encontram-se os modelos desenvolvidos para outros ecossistemas, tais como: Cerrados (Delitti \& Meguro 1984, Castro \& Kauffman 1998, Abdala et al. 1998, Delitti et al. 2006), Caatinga (Sampaio \& Silva 2005), Mata Mesófila Semidecídua Ciliar (Moreira-Burger \& Delitti 1999), Manguezal (Soares \& Novelli 2005) e Mata Atlântica (Burger \& Delitti, 2008).

O litoral do Estado de São Paulo apresenta remanescentes das formações florestais que cobriam os cordões arenosos da planície costeira, conhecidos como florestas de restinga. A urbanização da Baixada Santista vem exercendo forte pressão sobre esses ecossistemas, naturalmente frágeis por sua instalação sobre solos arenosos, muito lixiviados e pobres em nutrientes (Silva et al. 1993). A fitomassa exerce um papel fundamental na manutenção desses ecossistemas ao armazenar nutrientes que são reciclados (Casagrande 2003), garantindo sua sustentabilidade. Aspectos da ciclagem de nutrientes em formações florestais de restinga foram avaliados por Rebelo (1994), Moraes et al. (1999) e Pires et al. (2006) No entanto, para o entendimento do funcionamento desses ecossistemas são necessários mais estudos (Araújo \& Henriques 1984), uma vez que as informações existentes são insuficientes.

Apesar do importante papel da biomassa como descritor dos ecossistemas, não há relatos da quantificação da biomassa de florestas de restinga por métodos diretos. Neste estudo, a obtenção de informações da fitomassa de floresta de restinga através do método destrutivo, foi viabilizada no âmbito do Programa de Conservação dos Recursos Naturais do processo de licenciamento ambiental de um projeto de ampliação do Porto de Santos. Assim, o objetivo deste trabalho foi desenvolver modelos preditores da fitomassa arbórea aérea da Floresta Baixa de Restinga.

\section{Material e métodos}

Área de estudo - A área de estudo está localizada no Município de Santos, Estado de São Paulo, em local destinado à construção do Terminal Portuário Embraport, situado no
Sítio Sandi, entre os Rios Sandi e Diana, $\left(23^{\circ} 55^{\prime} 28^{\prime \prime}\right.$ S, $46^{\circ} 19^{\prime} 17,4^{\prime \prime} \mathrm{W}$ e $\left.23^{\circ} 54^{\prime} 23,4^{\prime \prime} \mathrm{S}, 46^{\circ} 18^{\prime} 23,7^{\prime \prime} \mathrm{W}\right)$.

A região apresenta clima quente e úmido. Segundo dados do Centro Integrado de Informações Agrometeorológicas do Instituto Agronômico de Campinas (CIIAGRO/IAC, 2008), no período de 2002 a 2007 a precipitação média anual foi de $1.993 \mathrm{~mm}$, sendo os meses de maio a setembro mais frios e menos úmidos, e de dezembro a março os meses mais quentes e mais úmidos. No período apresentado, em média, o mês mais quente foi fevereiro $\left(26,7^{\circ} \mathrm{C}\right)$ e o mais frio foi julho $\left(19,7^{\circ} \mathrm{C}\right)$. O mês de maior precipitação foi março $(271,3 \mathrm{~mm})$ e junho o mais seco $(48,5 \mathrm{~mm})$.

$\mathrm{Na}$ Baixada Santista, as florestas de restinga encontram-se bastante alteradas por desmatamentos, extração de areia, exposição à poluição industrial e urbanização (Silva et al. 1993, Rodrigues 2000). Para instalação do Terminal Portuário Embraport foi autorizado o desmatamento de uma área de 8,8 ha ocupados por vegetação classificada como Floresta Baixa de Restinga, em diferentes estágios de conservação (MKR \& Embraport 2005).

$\mathrm{Na}$ área estudada, pequenas manchas dessa formação florestal foram preservadas devido ao difícil acesso, uma vez que são rodeadas por campos úmidos e mangues, formando ilhas de vegetação arbórea. Um levantamento da história do uso e ocupação dessas áreas indica que permanecem em estado semelhante ao atual há mais de quarenta anos (MKR \& Embraport 2003). Estudos anteriores indicaram que os remanescentes florestais encontram-se em estágio médio e avançado de desenvolvimento e evidenciaram sua baixa riqueza em espécies e baixa diversidade, quando comparada a outras formações vegetais similares (MKR \& Embraport 2005).

Variações nas características do solo da área influenciam a estrutura da floresta. Esta se apresenta mais desenvolvida sobre manchas de solo areno-argiloso, bastante úmidos, com árvores que chegam aos $15 \mathrm{~m}$ de altura. Nelas a vegetação tem maior densidade das copas das árvores, gerando sombreamento nos estratos inferiores e acúmulo de serapilheira. Em áreas de solo predominantemente arenoso, a vegetação atinge um porte menor, raramente ultrapassando os $10 \mathrm{~m}$ de altura. As árvores formam copas menos densas permitindo maior penetração de luz e favorecendo o crescimento de espécies heliófilas no sub-bosque (MKR \& Embraport 2005).

Dentre as espécies ocorrentes na área de estudo, destacase o jerivá (Syagrus romanzoffiana), formando grandes agrupamentos nos fragmentos florestais, com indivíduos jovens a adultos, os quais podem atingir cerca de $15 \mathrm{~m}$ de altura. Neste estudo foi considerada uma espécie de especial interesse, pois foi identificada como uma espécie de grande relevância, segundo o valor de importância, no estudo da estrutura da vegetação, previamente realizado (MKR \& Embraport 2005). Além disso, é uma espécie bastante comum na região e de importância para a comunidade local, que a utiliza como planta ornamental, de fácil transplante mesmo quando adulta. 
Amostragem e análise de dados - A amostragem foi baseada na distribuição da vegetação em classes de diâmetro (DAP) apresentada por MKR \& Embraport (2005), garantindo a coleta de árvores de todos os tamanhos para a construção dos modelos (Overman et al. 1994). Percorrendo-se os 8,8 ha de Floresta Baixa de Restinga, foram selecionadas 204 árvores, sendo 102 indivíduos de Syagrus romanzoffiana (Cham.) Glassman. e as demais 102 de diferentes espécies, proporcionalmente distribuídas entre as classes de diâmetro.

As árvores selecionadas foram amostradas por método destrutivo (Whittaker et al. 1974, Chapman 1976, Golley et al. 1978). Destas foram tomadas medidas de circunferência à altura do peito $(1,3 \mathrm{~m})$, com fita métrica comum, e de altura total, com trena, após sua derrubada. As árvores foram cortadas a alguns centímetros do solo, utilizando-se moto-serra. Para a medida da massa, cada árvore foi dividida nos seguintes compartimentos: folhas, ramos finos (com diâmetro menor que 2,5 cm), galhos (com diâmetro igual ou maior que $2,5 \mathrm{~cm}$ ) e tronco. As folhas foram separadas do lenho manualmente e a massa úmida de cada porção medida com dinamômetro, com capacidade de $100 \mathrm{~kg}$ e precisão de 500 g. De cada árvore foi separada uma subamostra de cada compartimento, exceto do tronco, do qual foi retirada uma amostra da base e outra da porção correspondente a altura de 1,3 m. Todo material foi devidamente identificado, e a massa foi medida utilizando-se balança digital portátil, com capacidade de $5 \mathrm{~kg}$ e precisão de $10 \mathrm{~g}$. Todas as subamostras recolhidas em campo foram levadas a laboratório. Estas foram secas em estufa $\left(60\right.$ a $\left.70^{\circ} \mathrm{C}\right)$, até massa constante, para determinação do seu conteúdo de água e posterior cálculo da massa seca $(\mathrm{kg})$ de cada árvore. Cada árvore foi identificada em campo ou através da análise de material botânico devidamente coletado e preparado para identificação posterior. A partir da identificação, a densidade da madeira $\left(\mathrm{g} \mathrm{cm}^{-3}\right)$ foi obtida na literatura (Thiébault 1965, IPT 1978, IPT 1983, Lorenzi 1992, Chave et al. 2006) para todos os indivíduos coletados.

Para desenvolver as equações de regressão para a estimativa da fitomassa aérea da Floresta Baixa de Restinga, foi utilizada a massa seca total (MS, $\mathrm{kg}$ ) como variável dependente e o diâmetro à altura do peito $(\mathrm{d}, \mathrm{cm})$, altura (h, m), o quadrado do diâmetro multiplicado pela altura $\left(d^{2} h\right.$, $\mathrm{cm}^{2} \mathrm{~m}$ ) e a densidade multiplicada pelo quadrado do diâmetro e pela altura $\left(\rho \mathrm{d}^{2} \mathrm{~h}\right)$ como variáveis independentes. Altura e diâmetro são informações facilmente obtidas em campo. Todas as variáveis têm sido amplamente utilizadas em modelos preditores de biomassa de vegetação arbórea de ecossistemas tropicais (Folster et al. 1976, Jordan \& Uhl 1978, Golley et al. 1978, Tanner 1980, Saldarriaga et al. 1988, Brown et al. 1989, Martinez-Yrizar et al. 1992, Scatena et al. 1993, Overman et al. 1994, Santos 1996, Moreira-Burger \& Delitti 1999, Chave et al. 2001, Chave et al. 2005, Delitti et al. 2006).

As variáveis foram inicialmente analisadas de forma descritiva, através do cálculo de médias, desvios padrão, valores máximos, valores mínimos e medianas. Foram elaborados diagramas de dispersão entre todas as variáveis e verificado que os dados se ajustam a função potência $(\mathrm{Y}=a \mathrm{X} b)$. Estes foram submetidos a transformação logarítmica, a fim de se obterem as estimativas dos parâmetros da função $(\ln \mathrm{Y}=\ln a+b \ln \mathrm{X})$. A transformação logarítmica estabiliza o efeito do aumento da variância da biomassa com o aumento do tamanho das árvores, isto é, diminui a heterocedasticidade (Zar 1974), possibilitando a utilização da análise de regressão linear. Foi feita a análise de correlação de Pearson entre as variáveis. A forte correlação entre elas (colinearidade) impossibilita a utilização de análise de regressão múltipla entre as variáveis estudadas. Assim sendo, foi utilizada a análise de regressão linear simples para a estimativa dos parâmetros dos modelos preditores de biomassa. Os modelos que apresentaram tendências na análise de resíduos foram excluídos. Os modelos mais adequados foram definidos utilizando os seguintes critérios de seleção: maiores coeficientes de determinação $\left(\mathrm{R}^{2}\right)$ e menores valores de erro padrão da estimativa $\left(s_{y x}\right)$.

Em todas as análises foi utilizado o nível de significância de 5\%. As análises estatísticas (análise descritiva, análise de correlação, análise de regressão linear) foram realizadas utilizando-se os pacotes estatísticos Statistica para Windows (versão 6.0) e Statistical Package for the Social Sciences (SPSS) para Windows (versão 8).

\section{Resultados}

As árvores coletadas para a elaboração dos modelos preditores da fitomassa representaram 30 espécies, pertencentes a 21 famílias (tabela 1). A distribuição das árvores amostradas nas diferentes classes de DAP é apresentada na figura 1.

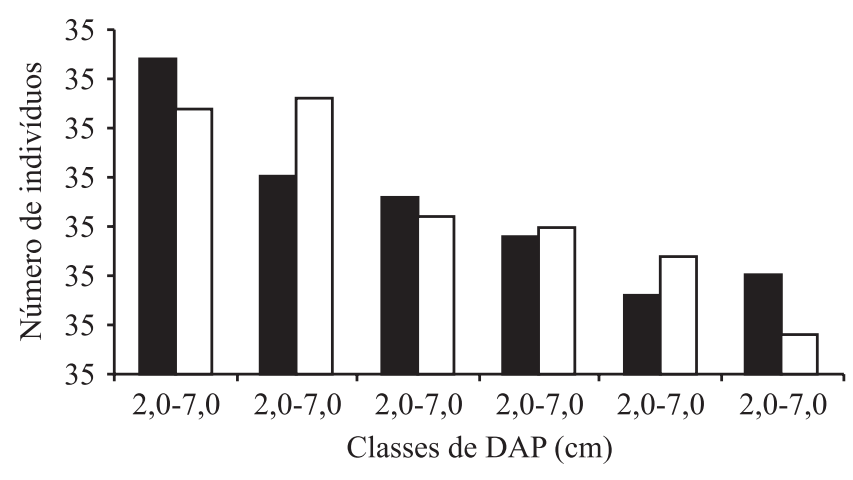

Figura 1. Distribuição de frequência nas classes de diâmetro (DAP) das árvores de diferentes espécies e de Syagrus romanzoffiana amostrados para elaboração dos modelos preditores de fitomassa da Floresta Baixa de Restinga. ( $\square=$ Syagrus romanzoffiana; $\mathbf{m}=$ diferentes espécies $)$.

Figure 1. Frequency distribution on diameter classes (DAP) of trees of different species and Syagrus romanzoffiana sampled to produce predictive models of phytomass for Restinga Forest. $(\square=$ Syagrus romanzoffiana; $\boldsymbol{\square}=$ different species $)$. 
Tabela 1. Espécies incluídas no estudo para elaboração de modelos preditores de fitomassa da Floresta Baixa de Restinga.

Table 1. Species included in the study for development of predictive models of Restinga Forest phytomass.

\begin{tabular}{|c|c|}
\hline Família & Espécie \\
\hline Anacardiaceae & Tapirira guianensis Aubl. \\
\hline Annonaceae & Annona cacans Warm. \\
\hline Aquifoliaceae & Ilex amara (Vell.) Loes. \\
\hline Arecaceae & $\begin{array}{l}\text { Syagrus romanzoffiana (Cham.) } \\
\text { Glassman }\end{array}$ \\
\hline Bignoniaceae & Tabebuia cassinoides DC. \\
\hline Celastraceae & Maytenus communis Reissek \\
\hline Celastraceae & Maytenus littoralis Carvalho-Okano \\
\hline Celastraceae & Maytenus robusta Reissek \\
\hline Clusiaceae & Calophyllum brasiliense Cambess. \\
\hline Euphorbiaceae & Sapium glandulatum Pax \\
\hline Fabaceae & $\begin{array}{l}\text { Abarema brachystachya (DC.) } \\
\text { Barneby \& J. W.Grimes }\end{array}$ \\
\hline Fabaceae & Andira fraxinifolia Benth. \\
\hline Fabaceae & Zollernia ilicifolia (Brongn.) Vogel \\
\hline Lauraceae & $\begin{array}{l}\text { Nectandra oppositifolia Nees \& } \\
\text { Mart. }\end{array}$ \\
\hline Melastomataceae & Tibouchina trichopoda Baill. \\
\hline Moraceae & Ficus insipida Willd. \\
\hline Myrsinaceae & Rapanea venosa (A. DC.) Mez. \\
\hline Myrtaceae & $\begin{array}{l}\text { Blepharocalyx salicifolius (Kunth) } \\
\text { O. Berg. }\end{array}$ \\
\hline Myrtaceae & Eugenia cf. umbelliflora O. Berg. \\
\hline Myrtaceae & Eugenia monosperma Vell. \\
\hline Myrtaceae & Marlierea tomentosa Cambess. \\
\hline Myrtaceae & Myrcia multiflora DC. \\
\hline Myrtaceae & Psidium cattleyanum Weinw. \\
\hline Nyctaginaceae & Guapira nitida (Mart.) Lundell \\
\hline Sapindaceae & Matayba elaeagnoides Radlk. \\
\hline Sapotaceae & $\begin{array}{l}\text { Manilkara subsericea (Mart.) } \\
\text { Dubard }\end{array}$ \\
\hline Solanaceae & Cestrum schlechtendalii G. Don \\
\hline Thymelaeaceae & Daphnopsis racemosa Griseb. \\
\hline Verbenaceae & Citharexylum myrianthum Cham. \\
\hline Viscaceae & $\begin{array}{l}\text { Phoradendron piperoides (Kunth) } \\
\text { Trel. }\end{array}$ \\
\hline
\end{tabular}

Modelos preditores da fitomassa da Floresta Baixa de Restinga, com exclusão do jerivá - Os modelos preditores da fitomassa de restinga foram elaborados a partir dos dados das 102 árvores de diferentes espécies, com exceção de Syagrus romanzoffiana, selecionadas na área de estudo. As árvores que compuseram a amostra apresentaram um DAP médio de $14,2 \mathrm{~cm}$, variando de $2,1 \mathrm{~cm}$ a $67,8 \mathrm{~cm}$ e altura média de $8,9 \mathrm{~m}$, variando de 1,8 a 19,5 m. Os parâmetros de distribuição das variáveis de estudo são apresentados na tabela 2. A análise de correlação linear de Pearson evidenciou que há colinearidade entre as variáveis independentes (tabela 3) inviabilizando a aplicação da análise de regressão múltipla na elaboração dos modelos. Os quatro modelos resultantes da análise de regressão foram estatisticamente significativos (tabela 4) e não apresentaram tendências

Tabela 2. Parâmetros de distribuição das variáveis de estudo utilizadas no desenvolvimento dos modelos preditores da fitomassa $(\mathrm{kg})$ acumulada pelas árvores da Floresta Baixa de Restinga, com exclusão do jerivá $(n=102)$. ( $n=$ número de árvores utilizadas no processo de modelagem; $\mathrm{dp}=$ desvio padrão; $\mathrm{d}=$ diâmetro $(\mathrm{cm})$; MS = massa seca $(\mathrm{kg}) ; \mathrm{h}=$ altura $(\mathrm{m}) ; \rho=$ densidade da madeira $\left.\left(\mathrm{g} \mathrm{cm}^{-3}\right)\right)$.

Table 2. Distribution parameters of the variables used in the study, used in the development of the predictive models of phytomass $(\mathrm{kg})$ accumulated by the trees of Restinga Forest, excluding jerivá $(n=102)$. $(n=$ number of trees used in the modeling process; $\mathrm{dp}=$ standard deviation; $\mathrm{d}=\operatorname{diameter}(\mathrm{cm})$; $\mathrm{MS}=\operatorname{dry}$ weight $(\mathrm{kg}) ; \mathrm{h}=$ height $(\mathrm{m}) ; \rho=$ wood density $\left(\mathrm{g} \mathrm{cm}^{-3}\right)$ ).

\begin{tabular}{lcccc}
\hline Variáveis & $\begin{array}{c}\text { Média } \\
(\mathrm{dp})\end{array}$ & Mínimo & Máximo & Mediana \\
\hline $\ln \mathrm{MS}$ & $3,4(1,6)$ & 0,4 & 7,9 & 3,5 \\
$\ln \mathrm{d}$ & $2,3(0,8)$ & 0,7 & 4,2 & 2,5 \\
$\ln \mathrm{h}$ & $2,1(0,5)$ & 0,6 & 3,0 & 2,1 \\
$\ln \mathrm{d}^{2} \mathrm{~h}$ & $6,8(2,0)$ & 3,0 & 11,3 & 7,1 \\
$\ln \rho \mathrm{d}^{2} \mathrm{~h}$ & $6,4(2,0)$ & 2,6 & 10,9 & 6,7 \\
\hline
\end{tabular}

Tabela 3. Matriz de correlação linear de Pearson entre as variáveis utilizadas no processo de modelagem para elaboração dos modelos preditores da fitomassa acumulada pelas árvores da Floresta Baixa de Restinga, com exclusão do jerivá. $(\mathrm{d}=$ diâmetro $(\mathrm{cm}) ; \mathrm{MS}=$ massa seca $(\mathrm{kg}) ; \mathrm{h}=$ altura (m); $\rho=$ densidade da madeira $\left.\left(\mathrm{g} \mathrm{cm}^{-3}\right)\right)$.

Table 3. Matrix of the Pearson's linear correlations among the variables used in the modeling process to produce predictive models of phytomass accumulated by trees in the Restinga Forest, excluding jerivá. $(\mathrm{d}=$ diameter $(\mathrm{cm}) ; \mathrm{MS}=$ dry weight $(\mathrm{kg}) ; \mathrm{h}=$ height $(\mathrm{m}) ; \rho=$ wood density $\left(\mathrm{g} \mathrm{cm}^{-3}\right)$ ).

\begin{tabular}{lccccc}
\hline Variáveis & $\ln \mathrm{MS}$ & $\ln \mathrm{d}$ & $\ln \mathrm{h}$ & $\ln \mathrm{d}^{2} \mathrm{~h}$ & $\ln \rho \mathrm{d}^{2} \mathrm{~h}$ \\
\hline $\ln \mathrm{MS}$ & 1,00 & 0,98 & 0,88 & 0,98 & 0,97 \\
$\ln \mathrm{d}$ & & 1,00 & 0,89 & 0,99 & 0,99 \\
$\ln \mathrm{h}$ & & & 1,00 & 0,94 & 0,93 \\
$\ln \mathrm{d}^{2} \mathrm{~h}$ & & & & 1,00 & 0,97 \\
$\ln \rho \mathrm{d}^{2} \mathrm{~h}$ & & & & & 1,00 \\
\hline$P<0,001$. & & & & &
\end{tabular}


Tabela 4. Descrição dos modelos preditores da fitomassa $(\mathrm{kg})$ acumulada pelas árvores da Floresta Baixa de Restinga, com exclusão do jerivá, resultantes da análise de regressão simples. A variável dependente é o logarítmo neperiano da massa seca. ( $a$ = constante da equação ou coeficiente linear da reta; $b=$ coeficiente de regressão; $d p=$ desvio padrão; IC = intervalo de confiança; $\mathrm{R}^{2}=$ coeficiente de determinação; estatística $F ; \mathrm{s}_{y x}=$ desvio padrão da estimativa).

Table 4. Description of the allometric models to estimate biomass $(\mathrm{kg})$ accumulated by the trees of the Restinga Forest, excluding jerivá, from simple regression analysis. The dependent variable is the natural logarithm of the dry mass. $(\mathrm{a}=$ constant of the equation or linear coefficient of the line; $\mathrm{b}=$ regression coefficient; $\mathrm{dp}=$ standard deviation; $\mathrm{IC}=$ confidence interval; $\mathrm{R}^{2}=$ coefficient of determination; $F$ statistic; $s_{y x}=$ standard error).

\begin{tabular}{lcccccr}
\hline variável preditora & $\mathrm{a}(\mathrm{dp})$ & $\mathrm{b}(\mathrm{dp})$ & $\mathrm{s}_{y x \%}$ & $\mathrm{IC}_{95 \%} \mathrm{~b}$ & $\mathrm{R}^{2}$ & $F$ \\
\hline $\ln \mathrm{d}(\mathrm{cm})$ & $-1,352(0,105)$ & $2,009(0,042)$ & 10,03 & {$[1,925 ; 2,093]$} & 0,957 & 2237,7 \\
$\ln \mathrm{h}(\mathrm{m})$ & $-3,150(0,352)$ & $3,119(0,164)$ & 22,62 & {$[2,793 ; 3,445]$} & 0,780 & 359,7 \\
$\ln \mathrm{d}^{2} \mathrm{~h}\left(\mathrm{~cm}^{2} \mathrm{~m}\right)$ & $-1,997(0,126)$ & $0,790(0,018)$ & 10,71 & {$[0,755 ; 0,826]$} & 0,951 & 1957,3 \\
$\ln \rho \mathrm{d}^{2} \mathrm{~h}\left(\mathrm{~g} \mathrm{~cm}^{-3}\right)$ & $-1,791(0,127)$ & $0,806(0,019)$ & 11,15 & {$[0,768 ; 0,843]$} & 0,947 & 1800,1 \\
\hline
\end{tabular}

$P<0,001$.

na distribuição dos resíduos (figura 2). Considerando os critérios estabelecidos para escolha do melhor modelo, foi selecionada a equação cuja variável preditora é o diâmetro a altura do peito (DAP). Tal modelo apresenta o maior coeficiente de determinação $\left(\mathrm{R}^{2}\right)$ e o menor erro padrão da estimativa $\left(s_{y x}\right)$.
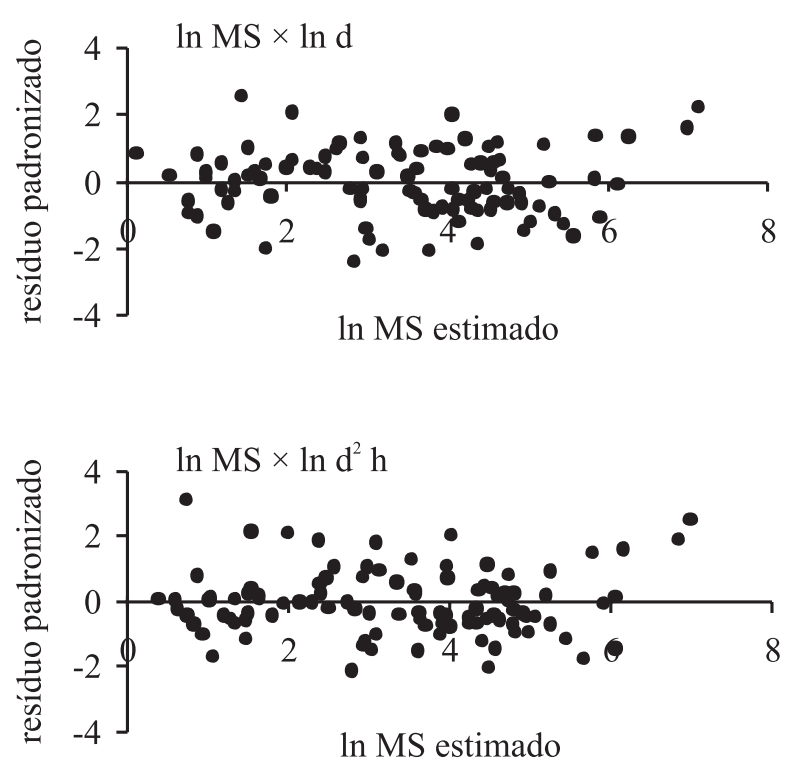

Modelos preditores da fitomassa de Syagrus romanzoffiana da Floresta Baixa de Restinga - Os modelos preditores da fitomassa do jerivá foram desenvolvidos com os dados de 102 árvores coletadas nas áreas de restinga. A altura média foi $5,8 \mathrm{~m}$, variando entre $1,3 \mathrm{~m}$ e $15,7 \mathrm{~m}$; o DAP médio foi de $13,1 \mathrm{~cm}$,
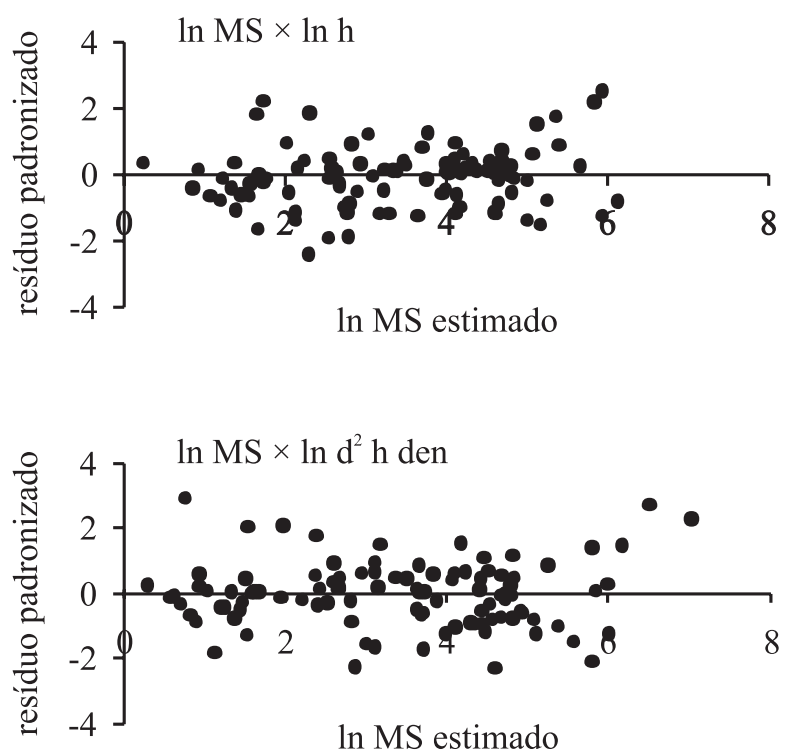

Figura 2. Diagramas de dispersão entre os resíduos padronizados e os valores ajustados dos modelos preditores da fitomassa (kg) acumulada pelas árvores da Floresta Baixa de Restinga, com exclusão do jerivá. (MS = massa seca (kg); $\mathrm{d}=$ diâmetro $(\mathrm{cm}) ; \mathrm{h}=$ altura $(\mathrm{m}) ;$ den $=$ densidade da madeira $\left.\left(\mathrm{g} \mathrm{cm}^{-3}\right)\right)$.

Figure 2. Diagrams of dispersion between the waste and the standardized values of the adjusted predictive models of phytomass $(\mathrm{kg})$ accumulated by the trees in the Forest of Restinga Baixa, excluding jerivá. $(\mathrm{d}=$ diameter $(\mathrm{cm}) ; \mathrm{MS}=$ dry weight $(\mathrm{kg})$; $\mathrm{h}=$ height $(\mathrm{m}) ;$ den $=$ wood density $\left.\left(\mathrm{g} \mathrm{cm}^{-3}\right)\right)$. 
variando entre $3,5 \mathrm{~cm}$ e $31,5 \mathrm{~cm}$. Os parâmetros de distribuição das variáveis utilizadas na modelagem são apresentados na tabela 5. Uma vez que entre as variáveis independentes há forte correlação (tabela 6) os modelos foram desenvolvidos por análise de regressão linear simples. Todos foram estatisticamente significativos (tabela 7) e não apresentaram vieses na análise de resíduos (figura 3). O modelo cuja variável preditora é o logarítmo neperiano da altura apresentou

Tabela 5. Parâmetros de distribuição das variáveis de estudo utilizadas no desenvolvimento dos modelos preditores da fitomassa $(\mathrm{kg})$ de Syagrus romanzoffiana $(n=102)$. ( $n=$ número de árvores utilizadas no processo de modelagem; $\mathrm{dp}=$ desvio padrão; $\mathrm{d}=$ diâmetro $(\mathrm{cm}) ; \mathrm{MS}=$ massa seca $(\mathrm{kg}) ; \mathrm{h}=\operatorname{altura}(\mathrm{m})$ ).

Table 5. Distribution parameters of the variables used in the study, used in the development of the predictive models of phytomass $(\mathrm{kg})$ accumulated by Syagrus romanzoffiana ( $n=102)$. ( $n=$ number of trees used in the modeling process; $\mathrm{dp}=$ standard deviation; $\mathrm{d}=$ diameter $(\mathrm{cm}) ; \mathrm{MS}=$ dry weight $(\mathrm{kg}) ; \mathrm{h}=$ height $(\mathrm{m})$ ).

\begin{tabular}{lcccc}
\hline Variáveis & $\begin{array}{c}\text { Média } \\
(\mathrm{dp})\end{array}$ & Mínimo & Máximo & Mediana \\
\hline $\ln \mathrm{MS}$ & $2,8(1,7)$ & 0,2 & 6,2 & 2,3 \\
$\ln \mathrm{d}$ & $2,4(0,6)$ & 1,3 & 3,5 & 2,4 \\
$\ln \mathrm{h}$ & $1,5(0,7)$ & 0,3 & 2,8 & 1,5 \\
$\ln \mathrm{d}^{2} \mathrm{~h}$ & $6,3(1,9)$ & 3,1 & 9,3 & 6,4 \\
\hline
\end{tabular}

Tabela 6. Matriz de correlação linear de Pearson entre as variáveis utilizadas no processo de modelagem para desenvolvimento dos modelos preditores de fitomassa Syagrus romanzoffiana.$(\mathrm{d}=$ diâmetro $(\mathrm{cm}) ; \mathrm{MS}=$ massa seca $(\mathrm{kg}) ; \mathrm{h}=$ altura $(\mathrm{m}))$.

Table 6. Matrix of the Pearson's linear correlations among the variables used in the modeling process to produce predictive models of phytomass accumulated by trees of Syagrus romanzoffiana. $(\mathrm{d}=$ diameter $(\mathrm{cm}) ; \mathrm{MS}=$ dry weight $(\mathrm{kg})$; $\mathrm{h}=$ height $(\mathrm{m})$ ).

\begin{tabular}{lcccc}
\hline variáveis & $\ln \mathrm{MS}$ & $\ln \mathrm{d}$ & $\ln \mathrm{h}$ & $\ln \mathrm{d}^{2} \mathrm{~h}$ \\
\hline $\ln \mathrm{MS}$ & 1,00 & 0,94 & 0,96 & 0,95 \\
$\ln \mathrm{d}$ & & 1,00 & 0,95 & 0,99 \\
$\ln \mathrm{h}$ & & & 1,00 & 0,98 \\
$\ln \mathrm{d}^{2} \mathrm{~h}$ & & & & 1,00 \\
\hline
\end{tabular}

$P<0,001$.
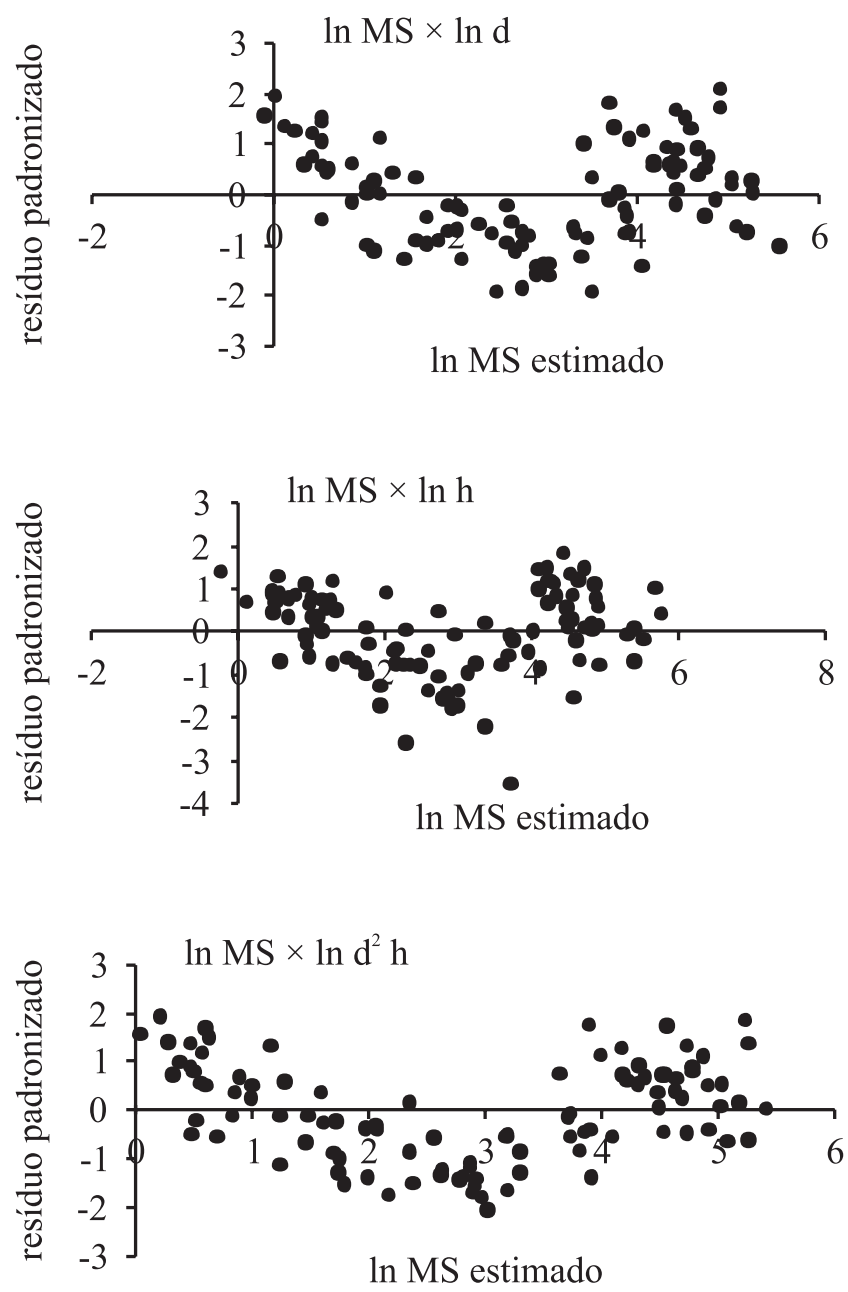

Figura 3. Diagramas de dispersão entre os resíduos padronizados e os valores ajustados dos modelos preditores da fitomassa $(\mathrm{kg})$ acumulada pelas árvores de Syagrus romanzoffiana.$(\mathrm{MS}=$ massa seca $(\mathrm{kg}) ; \mathrm{d}=$ diâmetro $(\mathrm{cm})$; $\mathrm{h}=\operatorname{altura}(\mathrm{m}))$.

Figure 3. Diagrams of dispersion between the waste and the standardized values of the adjusted predictive models of phytomass $(\mathrm{kg})$ accumulated by the trees of Syagrus romanzoffiana. $(\mathrm{d}=$ diameter $(\mathrm{cm}) ; \mathrm{MS}=$ dry weight $(\mathrm{kg})$; $\mathrm{h}=$ height $(\mathrm{m})$ ).

o maior coeficiente de determinação $\left(\mathrm{R}^{2}\right)$ e o menor erro padrão da estimativa $\left(s_{y x}\right)$, sendo considerado o mais adequado para a estimativa de fitomassa do jerivá.

Modelos preditores da fitomassa da Floresta Baixa de Restinga, com inclusão do jerivá - Numa etapa final do trabalho, foram elaborados modelos preditores de fitomassa para a comunidade como um todo, incluindo indivíduos de Syagrus romanzoffiana. A frequência 
Tabela 7. Descrição dos modelos preditores da fitomassa (kg) acumulada pelos indivíduos de Syagrus romanzoffiana, resultantes da análise de regressão simples. A variável dependente é o logarítmo neperiano da massa seca $(\mathrm{kg})$. ( $\mathrm{a}=$ constante da equação ou coeficiente linear da reta; $\mathrm{b}=$ coeficiente de regressão; $\mathrm{dp}=$ desvio padrão; $\mathrm{IC}=$ intervalo de confiança; $\mathrm{R}^{2}=$ coeficiente de determinação; estatística $F ; s_{y x}=$ desvio padrão da estimativa).

Table 7. Description of the allometric models to estimate biomass $(\mathrm{kg})$ accumulated by the trees of Syagrus romanzoffiana, from simple regression analysis. The dependent variable is the natural logarithm of the dry mass $(\mathrm{kg})$. $(\mathrm{a}=$ constant of the equation or linear coefficient of the line; $\mathrm{b}=$ regression coefficient; $\mathrm{dp}=$ standard deviation; $\mathrm{IC}=$ confidence interval; $\mathrm{R}^{2}=$ coefficient of determination; $F$ statistic; $s_{y x}=$ standard error).

\begin{tabular}{lcccccr}
\hline Variável preditora & $\mathrm{a}(\mathrm{dp})$ & $\mathrm{b}(\mathrm{dp})$ & $\mathrm{s}_{y x \%}$ & $\mathrm{IC}_{95 \%} \mathrm{~b}$ & $\mathrm{R}^{2}$ & $F$ \\
\hline $\ln \mathrm{d}(\mathrm{cm})$ & $-3,328(0,240)$ & $2,576(0,097)$ & 21,43 & {$[2,383 ; 2,769]$} & 0,874 & 700,879 \\
$\ln \mathrm{h}(\mathrm{m})$ & $-0,884(0,122)$ & $2,40(0,072)$ & 17,50 & {$[2,256 ; 2,544]$} & 0,916 & 1099,277 \\
$\ln \mathrm{d}^{2} \mathrm{~h}\left(\mathrm{~cm}^{2} \mathrm{~m}\right)$ & $-2,611(0,177)$ & $0,860(0,027)$ & 18,00 & {$[0,807 ; 0,913]$} & 0,911 & 1035,612 \\
\hline
\end{tabular}

$P<0,001$.

relativa da espécie na área de estudo é de 17,8\% (MKR \& Embraport 2005). Assim, para compor a amostra foram incluídos dados de 22 palmeiras na amostra de 102 árvores de diferentes espécies, proporcionalmente distribuídas nas classes de diâmetro. A amostra final foi composta por 124 árvores, com altura variando entre $1,8 \mathrm{~m}$ e $19,5 \mathrm{~m}$, com média de $8,7 \mathrm{~m}$, e o DAP variando entre $2,1 \mathrm{~cm}$ e $67,8 \mathrm{~cm}$, com média de $14,5 \mathrm{~cm}$. Os parâmetros de distribuição das variáveis utilizadas na modelagem são apresentados na tabela 8. Entre as variáveis independentes há forte correlação linear de Pearson (tabela 9). Os modelos resultantes da análise de regressão linear simples foram estatisticamente significativos (tabela 10) e apresentam boa distribuição dos resíduos (figura 4). Atendendo aos critérios de seleção pré-estabelecidos, o modelo selecionado foi aquele cuja variável preditora é o quadrado do diâmetro multiplicado pela altura.
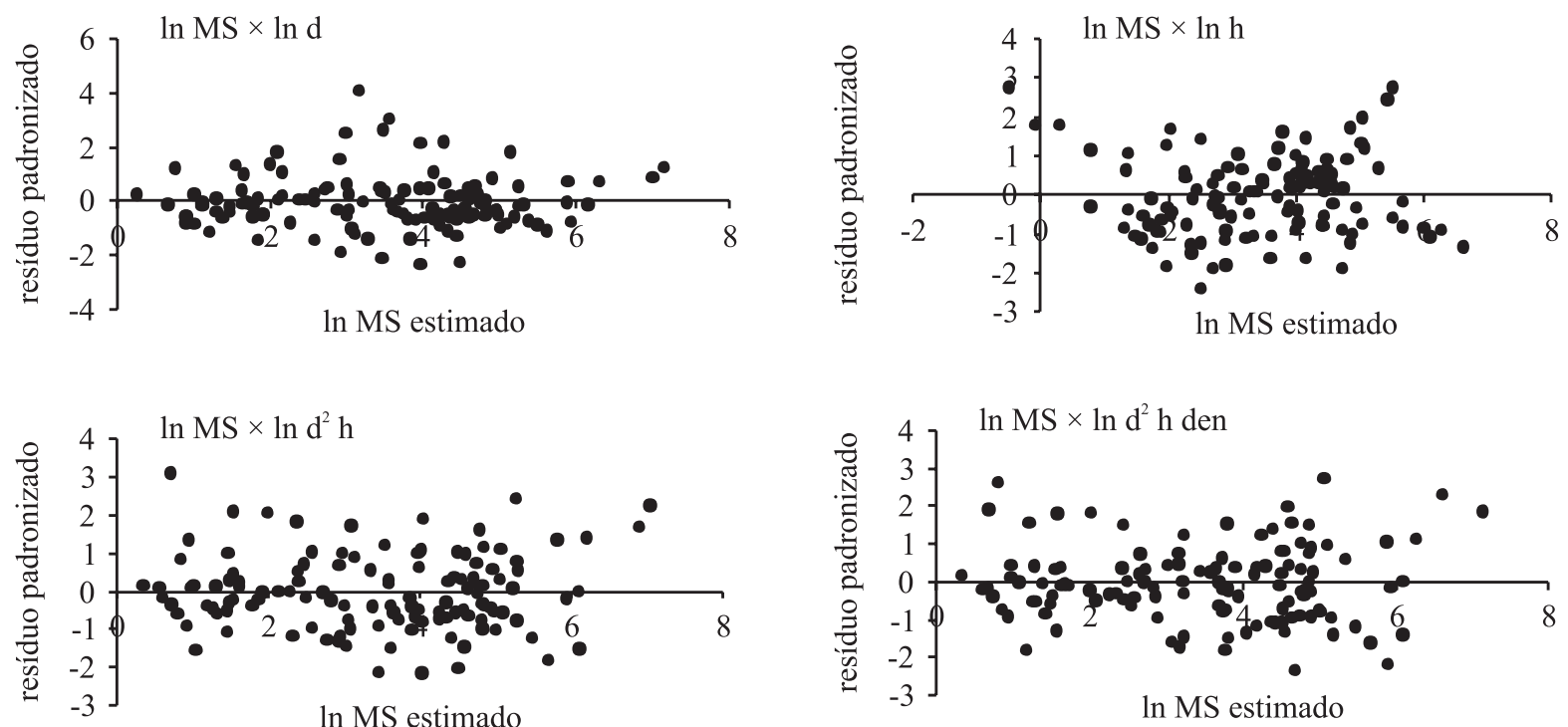

Figura 4. Diagramas de dispersão entre os resíduos padronizados e os valores ajustados dos modelos preditores da fitomassa $(\mathrm{kg})$ acumulada pelas árvores da Floresta Baixa de Restinga, com inclusão do jerivá. (MS = massa seca $(\mathrm{kg})$; $\mathrm{d}=\mathrm{diâmetro}$ $(\mathrm{cm}) ; \mathrm{h}=$ altura $(\mathrm{m}) ;$ den $=$ densidade da madeira $\left.\left(\mathrm{g} \mathrm{cm}^{-3}\right)\right)$.

Figure 4. Diagrams of dispersion between the waste and the standardized values of the adjusted predictive models of phytomass $(\mathrm{kg})$ accumulated by the trees in the Forest of Restinga Baixa, including jerivá. $(\mathrm{d}=$ diameter $(\mathrm{cm})$; $\mathrm{MS}=\mathrm{dry}$ weight $(\mathrm{kg})$; $\mathrm{h}=$ height $(\mathrm{m})$; den $=$ wood density $\left.\left(\mathrm{g} \mathrm{cm}^{-3}\right)\right)$. 
Tabela 8. Parâmetros de distribuição das variáveis de estudo utilizadas no desenvolvimento dos modelos preditores da fitomassa $(\mathrm{kg})$ acumulada pelas árvores da Floresta Baixa de Restinga, com inclusão do jerivá $(n=124)$. ( $n=$ número de árvores utilizadas no processo de modelagem; $\mathrm{dp}=$ desvio padrão; $\mathrm{d}=$ diâmetro $(\mathrm{cm}) ; \mathrm{MS}=$ massa seca $(\mathrm{kg}) ; \mathrm{h}=$ altura $(\mathrm{m}) ; \rho=$ densidade da madeira $\left.\left(\mathrm{g} \mathrm{cm}^{-3}\right)\right)$.

Table 8. Distribution parameters of the variables used in the study, used in the development of the predictive models of phytomass $(\mathrm{kg})$ accumulated by the trees of Restinga Forest, including jerivá $(n=124)$. $(n=$ number of trees used in the modeling process; $\mathrm{dp}=$ standard deviation; $\mathrm{d}=\operatorname{diameter}(\mathrm{cm})$; $\mathrm{MS}=\operatorname{dry}$ weight $\left.(\mathrm{kg}) ; \mathrm{h}=\operatorname{height}(\mathrm{m}) ; \rho=\operatorname{wood} \operatorname{density}\left(\mathrm{g} \mathrm{cm}^{-3}\right)\right)$.

\begin{tabular}{lccrc}
\hline Variáveis & $\begin{array}{c}\text { Média } \\
(\mathrm{dp})\end{array}$ & Mínimo & Máximo & Mediana \\
\hline $\ln \mathrm{MS}$ & $3,4(1,6)$ & 0,4 & 7,9 & 3,6 \\
$\ln \mathrm{d}$ & $2,3(0,8)$ & 0,7 & 4,2 & 2,4 \\
$\ln \mathrm{h}$ & $2,1(0,5)$ & 0,6 & 3,3 & 2,2 \\
$\ln \mathrm{d}^{2} \mathrm{~h}$ & $6,9(2,0)$ & 3,0 & 11,3 & 7,1 \\
$\ln \rho \mathrm{d}^{2} \mathrm{~h}$ & $6,4(1,9)$ & 2,6 & 10,9 & 6,8 \\
\hline
\end{tabular}

Tabela 9. Matriz de correlação linear de Pearson entre as variáveis utilizadas no processo de modelagem para elaboração dos modelos preditores da fitomassa acumulada pelas árvores da Floresta Baixa de Restinga, com inclusão do jerivá. $(\mathrm{d}=$ diâmetro $(\mathrm{cm}) ; \mathrm{MS}=$ massa seca $(\mathrm{kg}) ; \mathrm{h}=$ altura $(\mathrm{m})$; $\rho=$ densidade da madeira $\left.\left(\mathrm{g} \mathrm{cm}^{-3}\right)\right)$.

Table 9. Matrix of the Pearson's linear correlations among the variables used in the modeling process to produce predictive models of phytomass accumulated by trees in the Restinga Forest, including jerivá. $(\mathrm{d}=$ diameter $(\mathrm{cm})$; MS = dry weight $(\mathrm{kg}) ; \mathrm{h}=$ height $(\mathrm{m}) ; \rho=$ wood density $\left.\left(\mathrm{g} \mathrm{cm}^{-3}\right)\right)$.

\begin{tabular}{lccccc}
\hline Variáveis & $\ln \mathrm{MS}$ & $\ln \mathrm{d}$ & $\ln \mathrm{h}$ & $\ln \mathrm{d}^{2} \mathrm{~h}$ & $\ln \rho \mathrm{d}^{2} \mathrm{~h}$ \\
\hline $\ln \mathrm{MS}$ & \multirow{2}{*}{1,00} & 0,94 & 0,85 & 0,97 & 0,97 \\
$\ln \mathrm{d}$ & & 1,00 & 0,73 & 0,96 & 0,96 \\
$\ln \mathrm{h}$ & & & 1,00 & 0,88 & 0,87 \\
$\ln \mathrm{d}^{2} \mathrm{~h}$ & & & & 1,00 & 0,99 \\
$\ln \rho \mathrm{d}^{2} \mathrm{~h}$ & & & & & 1,00 \\
\hline$P<0,001$. & & & & & \\
\end{tabular}

Tabela 10. Descrição dos modelos preditores da fitomassa (kg) acumulada pelas árvores da Floresta Baixa de Restinga, com inclusão do jerivá, resultantes da análise de regressão simples. A variável dependente é o logarítmo neperiano da massa seca. $(a=$ constante da equação ou coeficiente linear da reta; $b=$ coeficiente de regressão; dp = desvio padrão; IC $=$ intervalo de confiança; $\mathrm{R}^{2}=$ coeficiente de determinação; estatística $F ; \mathrm{s}_{y x}=$ desvio padrão da estimativa).

Table 10. Description of the allometric models to estimate biomass $(\mathrm{kg})$ accumulated by the trees of the Restinga Forest, including jerivá, from simple regression analysis. The dependent variable is the natural logarithm of the dry mass. (a = constant of the equation or linear coefficient of the line; $b=$ regression coefficient; $d p=$ standard deviation; IC = confidence interval; $\mathrm{R}^{2}=$ coefficient of determination; $F$ statistic; $s_{y x}=$ standard error).

\begin{tabular}{|c|c|c|c|c|c|c|}
\hline Variável preditora & $\begin{array}{c}\mathrm{a} \\
(\mathrm{dp})\end{array}$ & $\begin{array}{c}\mathrm{b} \\
(\mathrm{dp})\end{array}$ & $\mathrm{S}_{y x \%}$ & $\mathrm{IC}_{95 \%} \mathrm{~b}$ & $\mathrm{R}^{2}$ & $F$ \\
\hline $\ln \mathrm{d}(\mathrm{cm})$ & $-1,221(0,165)$ & $1,990(0,067)$ & 16,68 & {$[1,858 ; 2,122]$} & 0,878 & 887,8 \\
\hline $\ln \mathrm{h}(\mathrm{m})$ & $-1,999(0,318)$ & $2,579(0,146)$ & 25,50 & {$[2,289 ; 2,869]$} & 0,715 & 310,2 \\
\hline $\ln \mathrm{d}^{2} \mathrm{~h}\left(\mathrm{~cm}^{2} \mathrm{~m}\right)$ & $-2,052(0,125)$ & $0,801(0,018)$ & 11,29 & {$[0,766 ; 0,835]$} & 0,944 & 2078,2 \\
\hline $\ln \rho \mathrm{d}^{2} \mathrm{~h}\left(\mathrm{~g} \mathrm{~cm}^{-3}\right)$ & $-1,780(0,126)$ & $0,814(0,019)$ & 11,88 & {$[0,777 ; 0,852]$} & 0,938 & 1872,1 \\
\hline
\end{tabular}

$P<0,001$.

\section{Discussão}

As árvores coletadas para a elaboração dos modelos preditores da fitomassa representaram todas as famílias encontradas em levantamento fitossociológico prévio (MKR \& Embraport 2005). Espécies como Syagrus romanzoffiana, Maytenus robusta, Rapanea venosa e Ilex amara consideradas de alto valor de importância no referido estudo, foram incluídas neste trabalho. Sendo espécies de ampla distribuição e comuns em diferentes fisionomias de restinga da região, contribuem para a adequação da aplicação dos modelos resultantes deste estudo.

O modelo cuja variável preditora é o diâmetro a altura do peito (DAP) foi considerado o mais adequado para estimar a fitomassa da Floresta Baixa de Restinga, com exclusão do jerivá. Porém, a inclusão da altura e da densidade no processo de modelagem também gerou 
modelos com elevados coeficientes de determinação, corroborando as observações feitas por outros autores para florestas tropicais (Chave et al. 2001). No entanto, neste trabalho considerou-se que o diâmetro (DAP), por ser uma informação facilmente obtida em campo e sempre presente nos inventários florestais, torna o modelo de fácil aplicação. Modelos desenvolvidos para diversas florestas tropicais também utilizam o DAP como variável independente (Golley et al. 1978, Tanner 1980, Brown et al. 1989, Martinez-Yrizar et al. 1992, Scatena et al. 1993, Overman et al. 1994, Higuchi et al. 1998, Chave et al. 2001, Burger \& Delitti, 2008), indicando sua ampla utilização em estudos dessa natureza. Já o melhor modelo para estimar a fitomassa aérea de Syagrus romanzoffiana da Floresta Baixa de Restinga foi aquele cuja variável preditora é a altura. Apesar de raros, os modelos desenvolvidos para palmeiras indicam que a altura explica a variação da quantidade de matéria orgânica estocada por essas árvores, como o observado por Vega et al. (2004) para a pupunheira (Bactris gasipaes Kunth). O modelo selecionado como preditor da fitomassa para a comunidade como um todo, incluindo indivíduos de Syagrus romanzoffiana, foi aquele cuja variável preditora é o quadrado do diâmetro multiplicado pela altura. Diversos são os autores que utilizam essa variável para a estimativa de fitomassa de florestas tropicais (Folster et al. 1976, Brown et al. 1989, Scatena et al. 1993, Overman et al. 1994, Burger \& Delitti, 2008), apesar da altura nem sempre estar disponível em levantamentos florestais. Assim, os resultados obtidos neste processo de modelagem evidenciam a influência do jerivá na determinação da melhor variável preditora da fitomassa da Floresta Baixa de Restinga. Considerando que a altura tem a maior correlação com a massa acumulada por essa palmeira, a inclusão dessa variável na modelagem faz com que o diâmetro sozinho deixe de explicar a maior proporção da variabilidade encontrada na fitomassa da floresta. Levando em conta a grande densidade do jerivá na área de estudo, a aplicação do modelo que o inclui deve produzir as melhores estimativas da fitomassa da vegetação de restinga em condições similares às do presente estudo. Apesar de todas as limitações impostas pelos estudos de natureza destrutiva como este, os modelos obtidos poderão ser utilizados nas estimativas da fitomassa das Florestas de Restinga em condições semelhantes às apresentadas neste trabalho.

Agradecimentos - Os autores agradecem a Embraport (Empresa Brasileira de Terminais Portuários S.A.) e a Consultoria Paulista de Estudos Ambientais Ltda (CPEA), pelo apoio logístico e a equipe composta por Alexandra
Helena Lisboa Boldrin, Bruno Pastrelli Kamada, Rodolfo Mulatinho Loero, Luiz Eduardo Guimarães Mariz, Ana Paula Schettino Moreira e Elaine Rovati, pelo trabalho de campo e discussão de resultados.

\section{Referências bibliográficas}

ABDALA, G.C., CALDAS, L.S., HARIDASAN, M. \& EITEN, G. 1998. Above and belowground organic matter and root shoot ratio in a cerrado in Central Brazil. Brazilian Journal of Ecology 2:11-23.

ARAÚJO, D.S.D. \& HENRIQUES, R.P.B. 1984. Análise florística das restingas do estado do Rio de Janeiro. In Restinga: origem, estrutura, processos (L.D. Lacerda, ed.).CEUFF, Niterói, p.159-93.

BROWN,S.,GILLESPIE,A.J.R.\&LUGO,A.E.1989.Biomass estimation methods for tropical forests with applications to forest inventory data. Forest Science 35:881-902.

BURGER, D. \& DELITTI, W.B.C. 2008. Allometric models for estimating the phytomass of a secondary Atlantic Forest area of southeastern Brazil. Biota Neotropica 8:131-136.

CASAGRANDE, J.C. 2003. Considerações sobre recuperação da fertilidade do solo para áreas degradadas. In Anais do Seminário temático sobre recuperação de áreas degradadas. (L.M. Barbosa \& A. Potomati, coord.). FAPESP/ IBt/SMA, São Paulo, p.92-93.

CASTRO, E.A. \& KAUFFMAN, J.B. 1998. Ecosystem structure in the Brazilian cerrado: a vegetation gradient of aboveground biomass, root biomass and consumption by fire. Journal of Tropical Ecology 14:263-283.

CHAPMAN, S.B. 1976. Production ecology and nutrient budgets. In Methods in plant ecology (S.B. Chapman, ed.). Blackwell, Oxford, p.157-228.

CHAVE, J., RIÉRA, B. \& DUBOIS, M. 2001. Estimation of biomass in a neotropical forest of French Guiana: spatial and temporal variability. Journal of Tropical Ecology 17:79-96.

CHAVE, J., ANDALO, C., BROWN, S., CAIRS, M.A., CHAMBERS, J.Q., EAMUS, D., FOLSTER, H., FROMARD, F., HIGUCHI, N., KIRA, T., LESCURE, J.P., NELSON, B.W., OGAWA, H., PUIG, H., RIÉRA, B. \& YAMAKURA, T. 2005. Tree allometry and improved estimation of carbon stoks and balance in tropical forests. Oecologia 145:87-99.

CHAVE, J., MULLER-LANDAU, H.C., BAKER, T.R., EASDALE, T.A., STEEGE, H.T. \& WEBB, C.O. 2006. Regional and phylogenetic variation of wood density across 2456 neotropical tree species. Ecological Applications 16:2356-2367.

CIIAGRO/IAC - Centro Integrado de Informações Agrometeorológicas do Instituto Agronômico de Campinas. 2008. Monitoramento Climatológico: Santos no período de 01/07/2002 até 31/12/2007. Disponível em http://www.ciiagro.sp.gov.br/ciiagroonline (acesso em 07/01/2008). 
DELITTI, W.B.C. \& MEGURO, M. 1984. Biomassa e mineralomassa do campo cerrado de Mogi-Guaçu, SP. Ciência e Cultura 6:612.

DELITTI, W.B.C., MEGURO, M. \& PAUSAS, J.G. 2006. Biomass and mineralomass estimates in a "cerrado" ecosystem. Revista Brasileira de Botânica 29:531-540.

FOLSTER, H., DE LAS SALAS, G. \& KHANNA, P. 1976. A tropical evergreen forest site with perched water table, Magdalena valley, Columbia. Biomass and bioelement inventory of primary and secondary vegetation. Oecologia Plantarum 11:297-320.

GOLLEY, F.B., MCGINNIS, J.T., CLEMENTS, R.G., CHILD, G.I. \& DUEVER, M.J. 1978. Ciclagem de minerais em um ecossistema de floresta tropical úmida. Edusp, São Paulo.

HIGUCHI, N., SANTOS, J., RIBEIRO, R.J., MINETTE, L. \& BIOT, Y. 1998. Biomassa da parte aérea da vegetação da Floresta Tropical Úmida de Terra-firme da Amazônia Brasileira. Acta Amazonica 28:153-166.

IPT - INSTITUTO DE PESQUISAS TECNOLÓGICAS. 1978. Fichas de características das madeiras brasileiras. IPT, São Paulo.

IPT - INSTITUTO DE PESQUISAS TECNOLÓGICAS. 1983. Manual de identificação das principais madeiras comerciais brasileiras. IPT/Promocet, São Paulo.

JORDAN, C.F.\& UHL, C. 1978. Biomass of a "tierra firme" forest of the Amazon Basin. Oecologia Pantarum 13:387-400.

KAUFFMAN, J.B., CUMMINGS, D.L. \& WARD, D.E. 1994. Relationship of fire, biomass and nutrient dynamics along a vegetation gradient in the Brazilian cerrado. Journal of Ecology 82:519-531.

KLINGE, H., RODRIGUES, W.A., BRUNIG, E. \& FITTKAU, E.J. 1975. Biomass and structure in a Central Amazonian rain forest. In Ecological studies: tropical ecological systems (F.B. Golley \& E. Medina, eds.). Springer Verlag, New York, p.115-122.

LORENZI, H. 1992. Árvores brasileiras. Plantarum, Nova Odessa.

MARTINEZ-YRIZAR, A., SARUKHAN, J., PEREZJIMENEZ, A., RINCON, E., MAASS, J.M., SOLIS-MAGALLANES, A. \& CERVANTES, L. 1992. Above-ground phytomass of a tropical deciduous forest on the coast of Jalisco, México. Journal of Tropical Ecology 8:87-96.

MCWILLIAN, A.L.C., ROBERTS, J.M., CABRAL, O.M.R., LEITÃO, M.V.B.R., COSTA, A.C.L., MAITELLI, G.T. \& ZAMPARONI, C.A.G.P. 1993. Leaf area index above-ground biomass of terra firme rain forest and adjacent clearings in Amazonia. Functional Ecology 7:310-317.

MKR \& EMBRAPORT. 2003.Terminal Portuário Embraport - Estudo de Impacto Ambiental. MKR/Embraport, São Paulo.
MKR \& EMBRAPORT. 2005. Complementação do diagnóstico da vegetação. Estudo de Impacto Ambiental. MKR/Embraport, São Paulo.

MORAES, R.M., DELITTI, W.B.C. \& STRUFALDI-DE VUONO, Y. 1999. Litterfall on litter nutrient content in two Brazilian Tropical Forests. Revista Brasileira de Botânica 22:9-16.

MOREIRA-BURGER, D. \& DELITTI, W.B.C. 1999. Fitomassa epigéa da mata ciliar do Rio Mogi-Guaçu, Itapira - SP. Revista Brasileira de Botânica 22: 429-435.

NELSON, B.W., MESQUITA, R., PEREIRA, J.L.G., SOUZA, S.G.A., BATISTA, G.T. \& COUTO, L.B. 1999. Allometric regressions for improved estimate of secondary forest biomass in the central Amazon. Forest Ecology and Management 117:149-167.

NOGUEIRA, E.M., FEARNSIDE, P.M., NELSON, B.W. \& FRANÇA, M.B. 2007. Wood density in forests of Brazil's 'arc of deforestation': Implications for biomass and flux of carbon from land-use change in Amazonia. Forest Ecology and Management 248:119-135.

NOGUEIRA, E.M., FEARNSIDE, P.M., NELSON, B.W., BARBOSA, R.I. \& KEIZER, E.W.H. 2008. Estimates of forest biomass in the Brazilian Amazon: New allometric equations and adjustments to biomass from woodvolume inventories. Forest Ecology and Management 256: $1853-1867$.

OVERMAN, J.P.M., WITTE, H.J.L. \& SALDARRIAGA, J.G. 1994. Evaluation of regression models for aboveground biomass determination in Amazon rainforest. Journal of Tropical Ecology 10:207-218.

PIRES, L.A., BRITEZ, R.M, MARTEL, G. \& PAGANO, S.N. 2006. Produção, acúmulo e decomposição da serapilheira em uma restinga da Ilha do Mel, Paranaguá, PR, Brasil. Acta Botanica Brasilica 20:173-184.

REBELO, C.F. 1994. Ciclagem de nutrientes minerais em floresta pluvial tropical de encosta e em floresta sobre restinga na ilha do Cardoso, Cananéia, São Paulo: decomposição da serapilheira. Dissertação de mestrado em Ecologia, Universidade de São Paulo, São Paulo.

ROCHOW, J.J. 1974. Estimates of above-ground biomass and primary productivity in a Missouri Forest. Journal of Ecology 62:567-577.

RODRIGUES, R.R. 2000. Recuperação de áreas degradadas em restinga. In Anais do workshop sobre recuperação de áreas degradadas da Serra do Mar e formações florestais litorâneas (L.M. Barbosa, coord.). SMA, São Paulo, p. $98-105$.

SALDARRIAGA, J.G., WEST, D.C., THARP, M.L. \& UHL, C. 1988. Long-term chronosequence of forest succession in the upper Rio Negro of Colombia and Venezuela. Journal of Ecology 76:938-958.

SAMPAIO, E.V.S.B. \& SILVA, G.C. 2005. Biomass equations for Brazilian semiarid caatinga plants. Acta Botanica Brasilica 19:935-943. 
SANTOS, J. 1996. Análise de modelos de regressão para estimar a fitomassa da floresta tropical úmida de terrrafirme da Amazônia brasileira. Tese de doutorado, Universidade Federal de Viçosa, Viçosa.

SCATENA, F.N., SILVER, W., SICCAMA, T., JOHNSON, A. \& SANCHEZ, M.J. 1993. Biomass and nutrient content of the Bisley Experimental Watersheds, Luquillo Experimental Forest, Puerto Rico, before and after Hurricane Hugo, 1989. Biotropica 25:15-27.

SILVA, I.X., MORAES, R.P., SANTOS, R.P., POMPÉIA, S.L. \& MARTINS, S.E. 1993. A degradação dos ecossistemas da Baixada Santista, São Paulo. p.129-140. In III Simpósio de Ecossistemas da Costa Brasileira. (S.Watanabe, coord.) ACIESP, Serra Negra.

SOARES, M.L.G. \& NOVELLI, Y.S. 2005. Above-ground biomass of mangrove species. I. Analysis of models.. Estuarine Coastal and Shelf Science 65:1-18.

TANNER, E.V.J. 1980. Studies on the biomass and productivity in a series of montane rain forests in Jamaica. Journal of Ecology 68:573-588.
THIÉBAULT, A. 1965. Nomenclature générale des bois tropicaux. Association Technique Internationale des Bois Tropicaux. Nogent-Sur-Marne.

UHL, C. \& JORDAN, C.F. 1984. Succession and nutrient dynamics following forest cutting and burning in Amazonia. Ecology 65:1476-1490.

UHL, C., BUSCHBACHER, R. \& SERRÃO, E.A.S. 1988. Abandoned pastures in eastern Amazonia. I. Patterns of plant succession. Journal of Ecology 76:663-681.

VEGA, F.V.A., BOVI, M.L.A., SPIERING, S.H. \& GODOY JÚNIOR, G. 2004. Relações alométricas para estimativa da fitomassa aérea em pupunheira. Horticultura Brasileira 22:104-108.

WHITTAKER, R.H., BORMANN, F.H., LIKENS, G.E \& SICCAMA, T.G. 1974. The Hubbard Brook ecosystem study: forest biomass and production. Ecological Monographs 44:233-252.

ZAR, J.H. 1974. Biostatistical analysis. Prentice Hall, Englewood Cliffs. 
